\title{
L'HOSPITALITÉ, LA CHARITÉ ET LE SOIN AUX MALADES À STAVELOT-MALMEDY AU MOYEN ÂGE (VII ${ }^{\mathrm{e}}$-XII ${ }^{\mathrm{e}}$ SIÈCLES)*
}

"Faites l'aumône et tout sera à votre disposition " ${ }^{1}$. Pour les moines, l'aide sociale est un fait quotidien, " une coutume de la vie monastique elle-même ${ }^{2}$, dont la motivation est religieuse. S'inscrivant dans la droite ligne de la charité chrélienne, tant de fois soulignée dans la Bible, et réaffirmée par la Règle de saint Benoît ${ }^{3}$, l'hospitalité, l'assistance aux pauvres et aux pèlerins font ainsi partie des devoirs d'une abbaye.

Au cour de l'Ardenne, saint Remacle fonda vers 650 Malmedy et Stavelot, respectivement sur les rives de la Warchenne et de l'Amblève $^{4}$. Pendant tout le Moyen Âge et jusqu'à la fin de l'Ancien Régime, Stavelot et Malmedy ont constitué une importante abbaye, royale puis impériale, groupant deux monastères géographiquement séparés, mais réunis sous la crosse d'un seul abbé. Une frontière diocésaine complique encore la situation: Stavelot relève du diocèse de

* Diesen Beitrag widmen wir in Dankbarkeit Herrn Dr. Ludwig Falkenstein, Aachen, Historisches Institut der Rheinisch-Westfälischen Technischen Hochschule.

1. Cartulaire de Gorze, cité par M. PARIsse, La vie religieuse en Lorraine au XI $I^{e}$ siècle, dans Sacris Erudiri, t. XX, 1971, p. 28. Le choix d'un texte de Gorze est ici volontaire puisque l'on doit à Odilon de Gorze la réforme de Stavelot-Malmedy vers 938 .

2. W. WitTers, Pauvres et pauvreté dans les coutumes monastiques du Moyen Âge, dans Études sur lhistoire de la pauvreté (Moyen Áge-XvI siécle), sous la direction de M. Mollat, Paris, 1974, p. 177-178.

3. Le devoir de l'hospitalité est inscrit dans la Règle de saint Benoît; le chapitre 53 est consacré à "La réception des hôtes ". Parmi les hòtes, "c'est aux pauvres et aux pèlerins surtout qu'on témoignera le plus d'attentions parce que c'est principalement en leur personne que l'on reçoit le Christ " (Regula Benedicti, c. 53, 15). A. de Vogüé, ("Honorer tous les hommes". Le șens de l'hospitalité bénédictine, dans A. DE VoGüé, Saint Benoit. Sa vie et sa règle. Eludes choisies, Bellefontaine, 1981 [Vie monastique, $\mathrm{n}^{\circ}$ 12], p. 121-131) analyse les directives spirituelles de saint Benoît en fait d'hospitalité, et ses sources. L'accueil dans l'hôtellerie sera surveillé par l'abbé, accompli avec cérémonial (lavement des mains et des pieds, verset psalmique) et confié à un frère.

4. Cfr notre article Saint Remacle, évangélisateur en Ardenne (ca. 650). Mythe et réalité, dans Bibliothéque de l'Instilut Historique Belge de Rome, Bruxelles-Rome, t. XXXVIII, 1996, p. 47-70. 
Liège, Malmedy ressortit à l'archevêché de Cologne ${ }^{5}$. D'une longue histoire se détachent très nettement deux abbatiats par leur importance: celui de Poppon (1020-1048) ${ }^{6}$ et celui de Wibald (1130-1158) ${ }^{7}$.

Grâce à l'abbaye, une charité active est présente au coeur de l'Ardenne. Trois "services" distincts sont accomplis par les moines: d'abord l'hospitalité aux hôtes de marque, ensuite l'accueil et l'assistance des moins favorisés, pauvres ou pèlerins, et enfin le soin aux malades 8 .

\section{L'hospitalité}

Les règlements de confraternité nous renseignent sur les aides réciproques accordées par les moines à leurs "confrères ${ }^{9}$; parmi ces avantages, l'hospitalité.

Avec Corvey (ca. 1147), si un frère est exclu d'une des deux abbayes à cause de ses fautes - propter vitia -, il sera accueilli materne dans l'autre maison jusqu'à la réconciliation. Avec Cornelimünster (11741192), un frère expulsé pourra trouver asile autant de temps qu'il le désirera ("accueil, hébergement, nourriture et toute charité »). Avec Prüm (1187), si un frère accusé d'une faute est exilé, il obtiendra aide et conseil - opem consilii et auxilii - dans l'autre monastère, qui lávorisera la réconciliation. Avec Saint-Hubert (1211), si un frère veut aller dans l'autre abbaye, avec une lettre de l'abbé, il y sera accueilli comme un fils; s'il est exilé pour ses fautes, de même il sera accepté jusqu'à ce qu'il obtienne le pardon.

5. Cfr les notices que nous avons consacrées dans le Lexikon des Mittelalters, Munich, à Malmedy, Poppon von Stablo, Quirinus von Malmedy, Remaclus, Sigebert III, Stavelot, et Wibald.

6. Pour toutes références, cfr notre article L'abbé Poppon (1020-1048) et son grand dessein pour Stavelot, dans Revue Mabillon, sous presse.

7. Cfr supra note 5, et le Catalogue de l'exposition Wibald, abbé de Stavelot-Malmedy et de Corvey 1130-1158, éd. J. STIEnnon \& J. Deckers, Stavelot, 1982.

8. L'hôpital au Moyen Âge est un refuge, un "hôtel " destiné à recevoir les hôtes. Il y eut des hôpitaux pour passants (pèlerins...), pour orphelins, pour vieillards, pour aveugles, et aussi bien entendu pour malades. Cfr l'excellente revue Annales de la Société Belge d'Histoire des Hopilaux, e. a. les articles de J.-L. DelatTre, Lhôpital monastique de Nivelles des origines à 1136, t. I, 1963, p. 7-17; P. BonenFant, Hôpitaux et bienfaisance publique dans les anciens Pays-Bas des origines à la fin du Xvilie siècle, t. III, 1965 , p. $1-44$, et A. Uytтebrouck, Hôpilaux pour lépreux ou couvents de lépreux. Réflexions sur le caractère des premieres grandes léproseries de nos régions à leurs origines, t. X, 1972, p. 5-29.

9. Notre article Les confraternités de l'abbaye de Stavelot-Malmedy, dans Bulletin de la Commission Royale d'Histoire, t. CLXI, 1995, p. 105-169, d'où sont extraits les exemples, de même la note suivante. 
Ainsi, un moine en rupture avec son abbaye peut trouver refuge à Stavelot-Malmedy selon des règles bien précisées, comme un moine de passage peut y obtenir gîte et couvert.

Le gîte est aussi un droit que l'abbaye détient dans ses possessions; il est rappelé lors de la cession de Germigny en 1160 à Saint-Remi de Reims ${ }^{10}$.

Plusieurs hôtes de marque visitèrent Stavelot, soit par nécessité, soit sur la route d'un voyage.

Vers 1033, Thierry de Leernes, futur abbé de Sainl-Hubert, quitte Lobbes et demande l'hospitalité à Poppon ${ }^{11}$. La solidarité des disciples de Richard de Saint-Vanne joue à fond ${ }^{12}$. Poppon réserve un accueil très cordial à son hôte pendant trois jours ${ }^{13}$.

D'après les Gesta abbatum Trudonensium, en 1114, en voyage pour Metz, Raoul de Saint-Trond reçut l'hospitalité (hospitium) à Stavelot où il participa aux fètes de commémoration de saint Remacle le 9 mai $^{14}$. Lors de la cérémonie, il se remémora les liens unissant Remacle et Trudon, patron de son abbaye, révélés par leurs Vitae respectives, et implora le secours du saint ardennais dans les problèmes qui l'opposaient à son avoué. Remacle exauça sa prière et Raoul obtint satisfac$\operatorname{tion}^{15}$.

Le 5 juin 1241, le rouleau funèbre de IIugues de Maumont, abbé de Solignac $(\dagger 1240)$, fut présenté à Stavelot par le porteur Jean ${ }^{16}$.

10. Porro quicquid tam in hominibus capitecensis quam in quibuslibet redditibus, hospitagiis, capitalibus, censibus, consuetudinibus quibuscumque, terris cultis et incultis, alienatum est et distractum a predicte ville territorio, universa hec perquirere et inventa possidere concessum est abbati et ecclesie $S$. Remigii (HR 252). Nous abrégeons sous cette forme "HR suivi du numéro de l'acte" les documents diplomatiques extraits du Recueil des chartes de l'abbaye de Stavelot-Malmedy, éd. J. Halkin \& C.-G. Roland, t. I, Bruxelles, 1909 et t. II, Bruxelles, 1930 (Commission Royale d'Histoire, Publications in- $4^{\circ}$ )

11. Vita Theoderici, éd. par W. Wattenbach, $M G H, S S$, t. XII, 1856, c. 10, p. 42. Sur ce texte, commentaire récent par J. Stiennon, A la table de Thierry $I^{e r}$, abbé de Saint-Hubert (1055-1086), dans Saint-Hubert d'Ardenne. Cahiers d'Histoire, t. VIII, Hommage a Léon Hannecart, Saint-Hubert, 1991, p. 275-284.

12. uno puero comitatus [...] [Theodericus] ad abbatem Popponem venit, qui et ipse $e$ numero discipulorum magistri sui fueral, multaque obedientia et ceterarum virtutum culmine promotus..., dans Vila Theoderici, op. cit., p. 42.

13. Cfr notre article Thierry de Leernes chez Poppon de Stavelot-Malmedy. Les réformateurs aux prises avec le diable, dans Malmedy Folklore, t. LVII, 1998, p. 75-86. Les faits nous sont rapportés par la Vita Theoderici abbatis Andaginensis, rédigée peu après la mort de Thierry I ${ }^{\text {er }}$, abbé de Saint-Hubert, en 1086 et achevée avant 1091.

14. Il s'agit du triomphe de saint Remacle (Liège, 9 mai 1071) et non de sa fête le 3 septembre, jour de sa mort.

15. Sur tout cet épisode, voir notre article sur les confraternités, op. cit., p. 148.

À propos de l'abbaye de Saint-Trond, à titre de comparaison, son hôpital est évoqué par J.-L. Charles, La ville de Saint-Trond au Moyen Age, Paris, 1965 (Bibliothèque de la Faculté de Philosophie \& Lettres de l'Université de Liège, Fasc. CLXXIII), p. 319.

16. Ibidem, p. 149. 
Une des grandes châsses mosanes présente, sur l'un de ses flancs, la réception légendaire de saint Hadelin, disciple de saint Remacle, par son maître à Stavelot. La scène d'orfèvrerie du milieu du xir ${ }^{\mathrm{e}}$ siècle, montre le saint s'inclinant respectueusement devant Remacle qui le bénit; trois jeunes moines, en coule et tonsurés, se tiennent debout derrière Remacle, devant un édifice censé être l'abbatiale de Stavelot ${ }^{17}$.

Les confraternités apportent un soutien essentiel dans la vie.

En visite à Solignac en 1134, Wibald avait renouvelé la fraternité au cours d'un chapitre présidé en commun avec son collègue l'abbé Maurice, après un sermon qui portait précisément sur la charité.

Cette grande "famille spirituelle " constituée entre les monastères est souvent due à l'ardeur, au dynamisme ou à la bonne volonté d'un abbé, initiateur des rapprochements; parmi ceux-ci, nous avions révélé l'action de Poppon, de Wibald et de son frère Erlebald (1158-1192).

Un autre genre d'hospitalité est le droit de procuration ou de gîte à l'égard de hautes personnalités. En 1147, lors du voyage du pape Eugène III dans l'Empire, une collecte fut organisée. Wibald, avec beaucoup d'empressement ${ }^{18}$, participa généreusement à ce servitium procurationis domni papae (HR 197).

\section{L'aumônerie monastique}

Benoît d'Aniane voulut uniformiser les usages monastiques par l'adoption exclusive et généralisée de la Règle de saint Benoît ${ }^{19}$. Dans

17. La légende commente la scène: VIRES DAT FAMULIS S(AN)C(T)I BENEDICTIO PATRIS (Catalogue de l'exposition: Trésors d'art religieux au pays de Visé, éd. P. \& M.-G. Bruyère-Boutier, Visé, 1988).

18. U. Berlière, Le droit de procuration ou de gîte. Papes et légats, dans Académie Royale de Belgique. Bulletin de la Classe des Lettres \& des Sciences Morales \& Politiques, 1919 , p. 511 et L. Falkenstein, Leistungsersuchen Alexanders III. aus dem ersten Jahrzehnt seines Pontifikates, dans Zeitschrift für Kirchengeschichte, t. CII, 1991, p. 61. Ce paiement de la procuratio canonica dans le cadre diocésain et la visite du synode diocésain par l'abbé montre que l'abbaye de Stavelot au xi ${ }^{\mathrm{e}}$ siècle était dépendante de l'évêque de Liège non seulement en vertu de la potestas ordinis, comme en témoignent les privilèges pontificaux, mais aussi dans tous les degrés de la juridiction ordinaire; sur ces problèmes qui sortent de notre propos, efr L. FALkENSTEIN, La papauté et les abbayes françaises aux $\mathrm{XI}^{e}$ et $\mathrm{X}_{1}{ }^{e}$ siècles. Exemption et protection apostolique, Paris, 1997 (Bibliothèque de l'École des Hautes-Études. Sciences Historiques \& Philologiques, t. CCCXXXVI).

19. "L'hospitalité, que la Regula veut voir exercée largement envers tous (53), se trouve restreinte par les Capitulaires d'Aix. Le $33^{\mathrm{e}}$ statut demande ut nullus plebius seu clericus secularis in monasterio ad habitandum recipiatur, nisi voluerit fieri monachus "(Ph. Schmitz, L'influence de saint Benoit d'Aniane dans l'histoire de l'ordre de Saint-Benoit, dans Atti di Settimane di Studio del Centro italiano di Studi sull'alto Medioevo, Spolète, t. IV, 1957, p. 412). 
les coutumiers monastiques, dès le $\mathrm{xx}^{\mathrm{e}}$ siècle, disparaît la "porta " pour faire place à deux offices distincts: l'hospilale pauperum dont s'occupe l'elemosinarius et auquel revient la dîme, et l'hospitale divitum dont s'occupe le custos hospitum et auquel revient la none.

Le Capitulare monasticum d'Aix-la-Chapelle du 10 juillet $817^{20}$ prévoit, auprès de chaque monastère, une " hostellerie " pourvue de tout le nécessaire pour l'hébergement des pauvres et des pèlerins, et le soin des malades de la communauté ${ }^{21}$. De tous les dons reçus, un dizième doit être donné aux pauvres.

Les $\mathrm{x}^{\mathrm{e}}$ et $\mathrm{xi}^{\mathrm{e}}$ siècles jettent sur les routes de nombreux êtres errants, qui circulent et ont besoin d'un gîte pour la nuit. Dans les monastères, un hospitium est toujours ouvert à ceux qui se présentent. "Le clerc ou le voyageur aisé - celui qui arrive à cheval — a parfois même la chance d'y trouver un quartier des hôtes, distinct de la chambre commune et pouilleuse où sont, malgré tout, heureux de s'étendre dans la plus grande promiscuité, les pauvres ${ }^{22}$. Ainsi le soin des pauperes $^{23}$ tend progressivement à se distinguer dans la réception des " hôtes ». À Reims ${ }^{24}$, Poppon était occupé au soin des pauvres in xenodochio ${ }^{25}$.

Les deux offices cités plus haut n'apparaissent pas dans les sources stavelotaines. En 922, un certain Liethfridus occupe la charge de por-

20. Capitularia regum Francorum, éd. A. Bonetıus, t. I, Hanovre, 1883, p. 343-349.

21. C. DE Clenco, La législation religieuse franque de Clovis à Charlemagne, t. II, Paris, 1936, p. 6-17.

22. P. Bonenfant, Les premiers " hospitia " de Bruxelles au XII siècle, dans Annales de la Société Belge d'Histoire des Hôpitaux, t. XI, 1973, p. 13.

La chronique d'Anselme de Liège rapporte que l'évêque Wazon (1042-1048), ami intime de Poppon, se faisait un devoir d'accueillir tous les étrangers qu'ils viennent ou non à cheval (Omnes peregrini supervenientes sine equis reciperentur; eos qui cum equis venissent recipere autumans episcopi interesse, MGH, SS, éd. R. KoEPKE, t. VII, 1846, p. 217).

23. Par facilité de langage, nous parlerons des "pauvres »; toutefois on tiendra compte des pertinentes remarques de J. Devisse, "Pauperes " et "paupertas 》 dans le monde carolingien. Ce qu'en dit Hincmar de Reims, dans Revue du Nord, t. XLVIII, 1966 , p. $273-287$.

24. Cifr infra notre commentaire, Vita Popponis, éd. W. Wattenbach, MGH, SS, t. XI, 1854 , p. 291-316, c. 8 .

25. Dans l'antiquité grecque, le terme désigne des chambres ou des maisons réservées aux hôtes. La forme grecque du mot conservée en latin prouve que l'institution est originaire des parties orientales de l'Empire romain. Il va être remplacé peu à peu, à partir du vil ${ }^{\mathrm{e}}$ siècle, par des équivalents d'origine latine tels que hospitium ou hospitale (W. Schönfeld, Die Xenodochiën in Italia und Frankreich im frühen Mittelalter, dans Zeitschrift der Savigny-Stiftung für Rechtsgeschichte, T. XLIII, 1922, Abt. XII, p. 18,54 ; E. LesNe, La propriété ecclésiastique en France aux époques romaine et mérovingienne, t. I, Lille-Paris, 1910, p. 401-412; J. ImBERT, Les hôpitaux en droit canonique, Paris, 1947, p. 46-50, et P. Bonenfant, Hôpitaux el bienfaisance publique, op. cit., p. 7). 
tarius, officier monastique chargé de recevoir les hôtes (HR 55). Des distributions quotidiennes ont généralement lieu, en nourriture, boisson et argent. Le portier reçoit les dìmes des produits et les répartit. La charité monastique concourt à cette redistribution des biens. C'est une charité organisée ${ }^{26}$. En 1127, lorsque la dîme de Sprimont est affectée à la réfection des moines et des pauvres, la répartition en est bien spécifiée: Unum modium frumenti dat portarius de frumento de Lovigeis, legumen et cervisam de communi fratrum prepositus, decem panes ad faciendas offas cellarius (HR 287).

L'accueil des pèlerins et des malades fait partie des missions des moines.

Les Miracula Remacli ${ }^{27}$ parlent du xenodochium, l'hospice du monastère; les pèlerins pauvres y sont généreusement accueillis et l'hospitalité consiste à leur fournir de la nourriture pendant presque huit jours alors qu'ils font leurs dévotions ${ }^{28}$.

Chaque année, le 24 juin, les habitants de la Famenne viennent à Stavelot pour y célébrer avec les moines la fête de saint Jean-Baptiste $^{29}$. Le lendemain, la prière des malades se poursuit à l'autel de saint Remacle et l'affluence est extraordinaire. Cette foule bruyante et envahissante perturbe la sérénité et le calme du monastère, au point qu'après l'office, les moines prolongent leur séjour au chœur pour y retrouver un peu de tranquillité ${ }^{30}$. Cette coutume est déjà ancienne au $\mathrm{x}^{\mathrm{e}}$ siècle quand sont rédigés les Miracles de saint Remacle ${ }^{31}$. À travers ces textes hagiographiques, on aperçoit tous ces malheureux étendus sur le pavé, affluant à Stavelot, leurs béquilles ou charrettes abandonnées devant la porte de l'abbatiale. Cette misère s'étale dans l'édifice avec toute sa détresse et surviennent des scènes surprenantes de guérison. Saint Remacle est l'intercesseur céleste et le "médecin " par excel-

26. R. GrégoIre, La place de la pauvreté dans la conception et la pratique de la vie monastique médiévale latine, dans Il monachesimo e la Riforma Ecclesiastica 1049-1122, Milan, 1971, p. 173-193.

27. Miracula sancti Remacli, éd. J. Veldius, dans AA.SS. Septembris, t. I, Anvers, 1746 , L. I, c. 28 , p. 702.

28. quibus de more in xenodochio exceptis, per dies ferme octo victui necessaria pietalis subrogamus, dans Miracula Remacli, op. cit., L. I, 28.

29. Mos namque regionis illius [...] continetur et praecipue ritu antiquo servatur, ut in Nativitate sancti Johannis Baptistae incolae ejus Patronum nostrum in multiplicibus commoditatibus saepe approbatum cum eulogiis suis orationis causa visitent, plus solito frequentent, alque etiam sollemnitatem insignis diei nobiscum concelebrent. Miracula Remacli, op. cit., L. II, 50. Secundum hanc insitam consuetudinem, L. II, 51.

30. Miracula Remacli, op. cit., L. II, 43, 50-53 et 64 .

31. Ritu antiquo, dans Miracula Remacli, op. cit., L. II, 50; secundum hanc insitam consuetudinem, L. II, 51. Il y aurait beaucoup à dire sur cette tradition mais cela sort du cadre de notre propos. 
lence $^{32}$. La Vie de saint Hadelin compare le pèlerinage de Stavelot à une ruche avec ses abeilles ${ }^{33}$.

A travers les donations faites ${ }^{34}$, on voit la foule des pauvres qui gravitent autour des monastères.

Une formule des diplômes de Childéric II (HR 8) et de Dagobert II (HR 9) indique que, non seulement les moines prieront pour la stabilité du royaume de leurs bienfaiteurs, mais aussi les pauvres qui viennent demander l'aumône aux monastères ${ }^{35}$.

Selon les coutumes de Fleury, le camérier remplit une bourse pour l'abbé lorsqu'il s'absente. «En effet, l'abbé ne doit jamais apparaître les mains vides lorsqu'il est à l'extérieur, mais il faut qu'il ait toujours la main prète à faire l'aumône. Et ce n'est pas en présence de tout un peuple ou devant ses serviteurs que l'abbé fait l'aumône à quelqu'un mais, conformément à l'Évangile, il s'efforce de faire la charité en secret et comme à la dérobée. Voici comment il fait: s'il se trouve en face d'une foule de pauvres, il s'arrête sur-le-champ et ne va pas plus loin avant de leur avoir donné sa bénédiction. Il ne fait pas l'aumône directement mais par l'intermédiaire de son chapelain et il ne reste pas à cheval pendant ce temps, mais, même si le chemin est boueux, aussitôt qu'il les a vus, sans retard, il descend humblement de cheval avec les frères qui l'accompagnent comme s'il s'agissait de recevoir le Christ. Et tandis que tout le monde s'agenouille, il donne sa bénédiction, puis ils remontent à cheval " ${ }^{36}$.

32. Expression utilisée dans la description des miracles du saint dans le Triumphus sancli Remacli de Malmundariensi coenobio, éd. W. Wattenbach, dans $M G H, S S$, t. XI, 1854, p. 433-461: L. II, c. 32: ut iure laudavit [mulier] laetissima medicum; L. II, c. 35: visitatus est [quidam clericellus] ab eodem medico circumstantium.

33. Catalogue de saint Hadelin, op. cit., p. 58 et 80. La même image se trouve dans les Miracula Remacli, op. cit., L. II, c. 44.

34. Sur les liens entre la commémoraison des morts et l'organisation de la charité, cfr L. Falkenstern, Die Kapellenstiftung zu Berensberg, die Pfarrei Laurensberg und die kirchliche Gebietsgliederung nördlich Aachens im Mittelalter, dans Kapelle und Kirche St.-Matthias zu Berensberg (1381-1890), t. I, Herzogenrath, 1991, p. 13.

35. vel pauperes qui ad ipsa loca sanclorum alimoniam expetunt, $\mathrm{HR} 8$ et 9.

36. Quelques usages de la vie quotidienne relevés dans la Vie de Jean de Gorze apparentent Grorze aux coutumes franques (à savoir le repos complémentaire après les vigiles, les trinae orationes et la visite des autels, les prières sub silentio et singillatim...). Les coutumes franques ont une tradition plus ancienne que Cluny. La comparaison de la Vita et des coutumes de Fleury par Dom Anselme Davril (L'abbaye de Gorze au $\mathrm{X}^{e}$ siécle. Études réunies par M. Parisse \& O.G. Oexle, Nancy, 1993, p. $183 \mathrm{sv}$.) fait apparaître aussi une grande austérité de vie: dureté de l'ascèse, repos complémentaire après les vigiles (propre à Jean de Gorze), multiplication des génuflexions et prostrations, usage restreint des bains (propre à Jean) el usage de la flagellation par les verges.

Les relations entre Metz, Toul et Trèves ont été étroites à cette époque et la date 
Au dire de son biographe, la réputation de charité ${ }^{37}$ de Poppon était telle que près de trois cents pauvres accouraient chaque premier jour du mois pour ètre nourris ${ }^{38}$. Longtemps, les monastères furent les seules institutions où les pauvres pouvaient trouver une aide. Lors de la cérémonie du Mandatum ${ }^{39}$, le Jeudi Saint, les moines lavaient les pieds et commémoraient le geste du Christ en nourrissant une centaine de pauvres $^{40}$.

Avec Richard de Saint-Vanne ${ }^{41}$, la prospérité matérielle permet la sérénité des cloîtres. Poppon en fut le fidèle disciple et l'héritier spiri-

rajeunie de fondation de Gorze (934) établit la simultanéité du mouvement réformateur dans cette région. Or on sait que les coutumes de Fleury (Consuetudines Floriacenses Antiquiores [abrégé $\mathrm{Th}$ ], rédigées au début du $\mathrm{xI}^{\mathrm{e}}$ siècle par Thierry d'Amorbach, moine à Fleury, éditées dans le Corpus consuetudinum monasticarum, t. VII-3 par A. Davril \& L. Donnat, Siegburg, 1984, p. 3-60) ont été apportées à Saint-Evre de Toul en 934. Mieux, une des recensions de la coutume de Saint-Emmcran est une proposition de coutumes pour un monastère en quête d'une " bonne " coutume et Dom Lin Donnat (L'abbaye de Gorze, op. cit., p. 180-181) émet l'hypothèse qu'elle est destinée à Gorze. Dom Anselme Davril, éditeur des coutumes de Fleury, en a donné une remarquable traduction sous le titre: La vie des moines en lan Mille (Saint-Benoît-sur-Loire, 1985, p. 23); cfr aussi A. DAvril, Un coutumier de Fleury au début du XI siècle, dans Rev. bén. t. LXXVI, 1966, p. 351-354, et Un moine de Fleury aux debuts de l'an mil: Thierry dit d'Amorbach, dans Etudes Ligériennes, Paris, 1975, p. 97-104.

37. Des références scripturaires renforcent la mise en exergue de la charité. Ainsi Oculus fui caeco et pes claudo. Pater eram pauperum... extrait du Livre de Job (c. 29, versets 15-16) cité au chapitre 28 de la Vita Popponis (Relevé aussi par P.-A. SigaL, Pauvreté et charité aux $X I^{e}$ et $X I I^{e}$ siècles d'après quelques textes hagiographiques, dans Mollat, Études, op. cit., 1974, p. 151) et les chapitres 31,32 et 17 cités au chapitre 8.

38. Vita Popponis, c. 28 p. 312 . Pierre De Spiegeler dans Les hôpitaux et l'assistance à Liège $\left(\mathrm{X}^{e}-\mathrm{X} v^{e}\right.$ siècles). Aspects institutionnels et sociaux, Paris, 1987, p. 43-44 (Bibliothèque de la Faculté de Philosophie \& Lettres, op. cil., Fasc. CCXLIX) replace cette action dans l'influence du mouvement de réforme monastique de Richard de SaintVanne dans tout le diocèse, et cite en comparaison l'abbé Thierry ${ }^{\text {er }}$ de Saint-Hubert (1055-1086) qui, en plus de l'aumône commune du monastère, nourrissait chaque jour douze pauvres auxquels il lavait les pieds et les mains.

39. La Règle de saint Benoît, comme nous l'avons dit, prescrivait à l'abbé et à la communauté monastique de laver les pieds des hôtes. Cette cérémonie du Mandé ou Mandatum revêtait une importance particulière le Jeudi Saint pour commémorer le geste accompli par le Christ envers ses apôtres, et était suivie d'une distribution d'aumônes (DE SPIEGELER, op. cit., p. 89-90).

40. Porro in Cena Domini antiqua constitutione recipiebantur LX pauperes ad Mandatum, quorum unusquisque haberet denarium unum, panem I el duo allecas cum legumine et biberem vini cum offis tribus. Additi sunt autem $X L$ pauperes ut centenarius numerus compleretur, quibus [...]. HR 287 (1127). Erlebald compléta la donation en 1182: ad complendum centenarium in Cena Domini pauperum numerum (HR 272).

41. Richard fut abbé de Saint-Vanne de Verdun de 1004 à 1046 . Sa forte personnalité, ses relations avec le monde aristocratique et notamment avec la Maison d'Ardenne, le soutien que lui procurèrent rois et empereurs, lui permirent d'exercer une action réformatrice sur de nombreux monastères, en Flandre d'abord, puis dans le diocèse de Liège. La réforme richardienne, c'est le retour à la ferveur monastique, le combat contre la tutelle laïque - en particulier les exactions des avoués — sur les abbayes, le tout dans l'esprit du mouvement pré-grégorien dont on connaît les liens avec la Lorraine. Alain Dierkens dans Abbayes et chapitres entre Sambre et Meuse 
tuel; il poursuivit ses relations avec lui après son départ de Verdun pour Stavelot. Selon la Vita Popponis, Richard écrivit à Poppon des lettres, consacrées notamment à la charité. La Vita rapporte qu'il les appréciait tellement qu'il souhaita qu'on les ensevelît avec lui après sa mort. Les moines de Stavelot eurent toutefois soin d'en prendre copie " afin que l'on pût voir l'affection et l'entente qui unissaient entre eux les deux abbés ${ }^{42}$; ainsi, tout qui s'intéresserait à la charité y pourrait trouver instruction ${ }^{43}$. Malheurcuscment, ces documents sont aujourd'hui perdus ${ }^{44}$.

La mort de Poppon est l'occasion pour son hagiographe de tracer un portrait du saint homme, très flatteur sans doute mais aussi très significatif de la ligne de conduite que s'était assignée le personnage ${ }^{45}$. Il loue sa componction, ce sentiment de tristesse devant l'indignité de l'homme à l'égard de Dieu qui incite souvent Poppon à pleurer abondamment: il passe jour et nuit en prière, s'agenouillant cent fois, et inonde de ses larmes le pavement; lorsqu'il célèbre la messe, il mouille aussi de ses pleurs la chasuble qu'il revêt. "Le pain des larmes est vraiment devenu sa nourriture ${ }^{46}$. Pour libérer son esprit de toute concupiscence, il s'inflige des mortifications, des jeûnes et des flagellations. Même très malade, il prend rarement des bains. Il s'abstient de tout mets gras. Il se mortifie dans sa chair et s'astreint à des pèlerinages à Rome, au tombeau des apôtres mais aussi d'autres saints. Sa charité est sans limite. Si tous les jours il nourrissait plusieurs pauvres, le premier du mois il en accueillait trois cents. Comme Job "qui était des

(VII $I^{e} I^{e}$ siècles). Contribution a l'histoire religieuse des campagnes du Haut Moyen Age, Sigmaringen, 1985 (Beihefte der Francia, t. XIV), p. 340-341 fait une synthèse de la réforme de Richard de Saint-Vanne, notamment d'après l'ouvrage de H. Dauphin, Le bienheureux Richard de Saint-Vanne de Verdun († 1046), Louvain-Paris, 1946; cfr aussi M. Parisse, La vie religieuse, op. cit., p. 11-38.

42. ut in eo amborum consensus el dilectio experitur, dans Vita Popponis, op. cit., c. 30 , et Dauphin, op. cit., p. 231.

43. quidque caritatis gratia valeat a legentibus ibi inveniretur, dans Vita Popponis, op. cil., c. 30 .

44. La Vie et les Miracles de saint Vanne, la Vie de saint Rouin sont les principales œuvres de Richard. Sur ce sujet, Dauphin, op. cit., p. 98 n. 2, et p. 231, 270, et 305.

45. Vita Popponis, op. cit., c. 28 . Nous espérons consacrer prochainement un article sur ce sujet.

46. U. BERLière, L'ascèse bénédictine des origines à la fin du XII siècle, Maredsous, 1927, p. 211. [...] cum psalmista dicere habebat: Fuerunt michi lacrimae meae panes die ac nocte, c. 28 , référence au Psaume 41, 4. Dom Berlière donne de nombreux exemples de moines en larmes quand ils priaient. D'après la Regula Benedicti (c. 20), la prière doit procéder d'un coeur purifié et ètre accompagnée des larmes de la componction. D'après Grégoire le Grand (Dialogues, II, 17), saint Benoît avait le don des larmes. Cette tradition bénédictine se poursuit avec Benoît d'Aniane, Jean de Gorze, Odilon de Cluny.... 
yeux pour l'aveugle et des pieds pour le boiteux », Poppon épousait la cause du pauvre. Il faisait aussi des dons en or et en argent aux ermites et les encourageait de la sorte dans leur vocation.

À Malmedy, l'abbé Folmar redistribue les revenus de l'aumônerie affectés à la subsistance des paurres ${ }^{47}$.

L'abbé Poppon II avait octroyé des revenus ad servitium fratrum et ad usus pauperum (HR 141); en 1140, Wibald règle l'aumône des pauvres lors de la célébration de l'anniversaire de Poppon $\mathrm{II}^{48}$. Dans la redistribution des biens confiés par Poppon II en bénéfice, Wibald agit encore ad usus fratrum et pauperum atque hospitium (HR 175).

\section{Le soin aux malades}

Le 13 avril 862 le souverain Lothaire II s'occupe de la répartition des biens ad hospitale ejusdem coenobii et ordonne que l'on paie à l'hôpital de l'abbaye absque negligentia et tarditate la dîme des fruits dans les manses seigneuriaux (HR 34). Cette ordonnance est confirmée le 10 juin 873 (HR 36) ${ }^{49}$.

Vers 1148, on apprend l'achèvement des travaux des bâtiments de l'infirmerie (HR 214); en 1140, Wibald avait déterminé les revenus qui lui étaicnt affectés (HR 289) ${ }^{50}$. En 1148, il demandail aux moines de destiner certains revenus vestris et pauperum usibus (HR 210). La donation de 1172 du duc de Limbourg-Arlon est faite ad sustentationem et usus pauperum quorum ibi maxima confluentia est ${ }^{51}$.

47. Cfr J. Stiennon, Le scriptorium el le domaine de labbaye de Malmedy du $X^{e}$ au début du XIII siècle d'après les manuscrits de la Bibliothèque Vaticane, dans le Bulletin de l'Institut Historique Belge de Rome, t. XXVI, 1950, p. 23.

48. et XXcim pauperes ad elemosinam simili et equali refectione quam habent fratres reficiantur, HR 289.

49. Cfr Fr. BaIx, Étude sur l'abbaye et principauté de Stavelot-Malmédy. Première partie: L'abbaye royale et bénédictine (Des origines à l'avènement de S. Poppon, 1021), Paris-Charleroi, 1924, p. 93 et 95. Cette disposition suppose aussi un fonds de biens distincts des biens communs de l'abbaye, comme nous l'a très aimablement fait remarquer $\mathrm{I}_{\text {.. Falkenstein. }}$

50. En 1563 sera consacrée la chapelle de l'infirmerie du monastère sous l'invocation des saints Jacques, Victor, Gengulphe, Cosme, Damien, Lazare et Madeleine (Cfr notre ouvrage Les reliques de Stavelot-Malmedy. Nouveaux documents, Malmedy, 1989, p. 78 et 106). On relèvera les noms de ces derniers saints. La gravure de G.-Chr. Kilian, vers 1730 (W. Legrand, Léglise abbatiale de Stavelot, dans Bulletin de la Société d'Art \& d'Histoire du Diocèse de Liège, t. XLIII, 1963, p. 194-195), situe les bâtiments de l'infirmerie.

51. Commentaires dans Fr.-J. JAkoBi, Wibald von Stablo und Corvey (1098-1158) Benediktinischerabt in frühen Stauferzeit, Münster, 1979 (Veröff. der hist. Kommission für Westfalen, t. X, Abhandl. zur Corveger Geschichtsschreibung, t. V ), p. 262. 
La maladie est à l'origine de la pauvreté: c'est elle qui entraîne l'incapacité de travail. À la lecture des Miracles de saint Remacle, l'église abbatiale, à en juger par le nombre important de malades qui s'y pressaient, devait souvent ressembler à un hôpital, au sens moderne du mot.

La lèpre existait en Occident avant les Croisades ${ }^{52}$. La Vita Popponis met son héros aux prises avec un lépreux à Saint-Thierry de Reims. Poppon y eut pour maître Eilbert - sagacis ingenii virum ${ }^{53}$-, le frère de Gérard de Rumigny, futur évêque de Cambrai ${ }^{54}$. Alors qu'Eilbert était portier, l'abbé confia à Poppon l'accueil et le soin des pauvres à l'hôtellerie: pro recipiendis in xenodochio pauperibus (c. 8). Il fut l'économe et distribua les vivres et les aumònes. Un jour, un lépreux se présenta. Poppon ne fut nullement horrifié à sa vue et s'activa à le réconforter. Comme la nuit froide arrivait, il se montra charitable - alter Martinus ${ }^{55}$ - envers le pauvre malade et le recouvrit de sa propre couverture. Le lépreux, emmitouflé dans ce vêtement, sua abondamment et la lèpre le quitta. Voulant cacher ce miracle ${ }^{\overline{6}}$, Poppon continua à se servir de cette couverture ${ }^{57}$. Il supplia Eilbert de n'en rien dire et seul son biographe en eut connaissance indirectement.

52. G. Kurth, La lèpre en Occident avant les Croisades, Paris, 1907, et Fr. BÉrict, Histoire des lépreux au Moyen Âge. Une société d'exclus, Paris, 1988, p. 151, qui écrit: «Si, contrairement à une légende tenace, accréditée, entre autres, par le talent de Michelet, la lèpre ne représente en rien un "sale résidu " des croisades, mais bel et bien une endémie présente dès les premiers siècles de notre ère, les léproseries restèrent fort rares jusqu'aux XI-XII siècles ». Cfr aussi G. PICHon, Essai sur la lepre du haut Moyen $\hat{A g e}$, dans Le Moyen Age, t. XC, 1984, p. 331-356 et compléments bibliographiques dans N. Bériou \& Fr.-Ol. TouAt, Voluntale Dei leprosus. Les lépreux entre conversion et exclusion aux XII et XIII siècles, Spolète, 1991 (Testi, Studi, Strumenti, n ${ }^{\circ} 4$ ), p. IX.

53. Vita Popponis, op. cit., c. 7.

54. Eilbert deviendra bientôt abbé de Maroilles (Marriliacensium postea abbate c. 7). S. Balau, Les sources de l'histoire de Liege au Moyen Äge, Bruxelles, 1903, p. 216. U. Berliere, Abbaye de Stavelot-Malmedy, dans Monasticon belge, t. II, Province de Liège, Maredsous, 1928, p. 78: Eilbert fut aussi abbé de Saint-André du Cateau.

55. Le c. 8 est exemplaire en citations et réminiscences bibliques et hagiographiques. Est cité Job, 31, 32: Aucun résident étranger ne passait la nuit dehors. Je laissais mes portes ouvertes sur le sentier; et 31, 17: Et [si] je mangeais seul mon morceau. Tandis que l'orphelin de père n'en mangeait pas.

56. Comme le Christ, il est en présence d'un lépreux et adopte une attitude sernblable de charité: imitans certe Dominum, qui pro humilitate et compassione leprosum, quem lex tangere vetuerat, tetigit et mundavit. Les exemples bibliques - les miracles du Christ - ou hagiographiques - saint Benoit, saint Martin... - sont bien présents dans les mentalités au Moyen Âge (Cfr M. Forrier, La lèpre à travers lespace et le temps, dans le Catalogue de l'exposition: La lèpre dans les Pays-Bas, XII ${ }^{e}-X v 1 I I^{e}$ siecles, Bruxelles, Archives Générales du Royaume, 1989, p. 18-22).

57. Proinde nemo Deum in sanctorum suorum vestimentis salutem consequi posse dubilel, quem ex vestimentorum suorum fimbriis mulierem sanguinis profluvio laborantem sanasse liquido patet, c. 8. La couverture devient une vraie relique; toutefois on n'en trouve aucune autre mention. On pense bien sûr à des exemples semblables comme 
À Malmedy existait une léproserie dont la chapelle ${ }^{58}$ dédiée à sainte Marie-Madeleine fut consacrée le 8 aoùt 1188 (HR 276) ${ }^{59}$. Plus de quarante noms de donateurs sont rappelés dans l'acte de 1188 (HR 276): certains lèguent un terrain, d'autres une rente en argent ou en blé ${ }^{60}$. C'est Erlebald, frère de Wibald, qui procède à la dédicace ${ }^{61}$. En 1179 , le troisième concile de Latran avait reconnu aux lépreux vivant en communauté le droit d'avoir un oratoire et un cimetière ${ }^{62}$.

À Stavelot, la chapelle Saint-Laurent, dont la dédicace remontant au 24 décembre 1030 fut renouvelée en $1246^{63}$, est aussi la chapelle d'une léproserie mais il n'est pas possible de savoir en quelle année celle-ci fut créée $^{64}$. Comme à Malmedy, elle est située hors de la ville, dans un endroit écarté, au-delà de l'Amblève.

La pauvreté est requise pour faire partie de l'ordo monachorum ${ }^{65}$, contrairement à l'ordo clericorum qui autorisait la propriété ${ }^{66}$. Le moine

le manteau de saint Martin ou celui de saint Jacques; cfr notre article Documents inédits sur le trésor des reliques des abbayes bénédictines de Saint-Laurent et de Saint-Jacques de Liege (XI ${ }^{e}-\mathrm{XVIII}{ }^{e}$ siecles), dans Bulletin de la Commission Royale d'Histoire, t. CLVIII, 1992, p. 1-49.

58. Capellam infirmorum Malmundariensium, domum et ecclesiam leprosorum Malmundariensium, HR 276.

59. J. Stiennon, Étude critique des deux premiers actes relatifs à la léproserie de Malmedy, dans Bulletin de la Commission Royale d'Histoire, t. CXV, 1950, p. 443-458, en réponse à l'article de J. Bastin, Deux léproseries antérieures aux Croisades, dans Actes de la Fédération Archéologique \& Historique de Belgique, Congrès de Malines, 1911, t. II, p. $339-342$.

60. On conserve des reliques des martyrs de Trèves provenant de cette chapelle, cfr notre ouvrage, p. 73. Signalons au passage que l'hospitium peregrinorum est mentionné à Malmedy au début du xvi siècle dans les Miracles de Saint Quirin (Cfr notre édition Les Miracles de saint Quirin de Malmedy, un livret hagiographique médiéval au cour du $X V l^{e}$ siècle, dans Bulletin de la Commission Royale d'Histoire, sous presse, Miracle $\mathrm{n}^{\circ}$ 1).

61. La chapelle a survécu jusqu'à nous, à travers l'époque moderne et le culte de Notre-Dame des Malades (Cfr J. Gelis, Les "sanctuaires à répit " dans les Ardennes belge et française, dans Catalogue de l'exposition: Trésors d'Ardenne. Art religieux \& croyances populaires en Ardenne \& Luxembourg, Bastogne, 1987, p. 55-64).

62. J. Avril, Le $I I I^{e}$ concile de Latran et les lépreux, dans Rebue Mabillon, t. LX, 1981, p. 21-76.

63. Notre ouvrage Les reliques, op. cit., p. 78 et $105-106$.

64. Les documents se regroupent autour de 1246-1248; l'abbé Bastin (op. cit., p. 341-342) suppose qu'en 1030 déjà la léproserie existait, mais nous serions moins affirmatif que lui.

65. "S'il [le novice] possède quelque avoir, ou bien il le distribuera auparavant aux pauvres, ou bien il l'attribuera au monastère par une donation solennelle, sans rien se réserver du tout; car il sait que, dès cet instant, il ne peut plus mème disposer de son propre corps " (Règle de saint Benoît, c. 58, 24-25). Faut-il rappeler que la pauvreté, avec l'obéissance et la chasteté, fait partie des vœux solennels monastiques?

66. J. SEMmLER, Le monachisme occidental du VIII au $\mathrm{X}^{e}$ siècle: formation et réformation, dans Le monachisme à Byzance et en Occident du VIII au $x^{e}$ siècle. Aspects internes et relations abec la société. Actes du Colloque de l'ULB, Bruxelles-Maredsous, 14-16 mai 1992, éd. A. Dierkens, D. Misonne \& J.-M. Santerre, dans Rev. bén., t. CiII, 1993, p. 74 et A. DE VoGüÉ, La pauvreté dans le monachisme occidental du $I V^{e}$ au $V_{I} I^{e}$ siècle, dans Collectanea Cisterciensia, t. XLVI, 1984, p. 177-185. 
renonce à tout bien personnel et à toute propriété privée. La pauvreté individuelle n'empêche pas la richesse collective de la communauté. Cette richesse est nécessaire pour organiser une " charité rituelle et codifiée ${ }^{67}$ qui nourrira les pauvres par des distributions fixes.

Liée comme elle l'est aux réformes monastiques (Benoît d'Aniane, Jean de Gorze, Richard de Saint-Vanne), la charité, au sens le plus large du terme, est une composante de la spiritualité monastique. Le moine assure une part de son salut par son souci des pauvres. Un idéal de perfection est proposé, qui conduit à la sainteté ${ }^{68}$. La charité occupe une place importante dans tout ce climat spirituel propre aux $\mathrm{xI}^{\mathrm{e}}$ et $\mathrm{xII}^{\mathrm{e}}$ siècles, face au "nouveau monachisme " et aux transformations de la vie religieuse ${ }^{69}$. Son action concrète sur le terrain ne peut faire oublier que cette vertu théologale se révèle aussi un baromètre de l'influence des réformes.

Toute pratique monastique subit une inévitable usure interne. À Stavelot-Malmedy, le phénomène fut accentué par la longue période des abbatiats laïques depuis le milieu du $\mathrm{Ix}^{\mathrm{e}}$ siècle $^{70}$. Un nécessaire renouveau devait se manifester. Encore faut-il se mettre d'accord sur la définition de "réforme monastique " ou de "restauration de monastère $~^{71}$. Deux facteurs principaux entrent en lice: le rétablissement des ressources matérielles du monastère et le retour à une vie régulière conforme à la Règle de saint Benoît. Ainsi sont inévitablement unies gestion du temporel et animation spirituelle. L'assurance du quotidien

67. Sigal, op. cit., p. 151.

68. La rédaction d'une Vita Popponis va dans ce sens, même si le culte de l'abbé ne se développera vraiment qu'au xvir ${ }^{\mathrm{e}}$ siècle. Cfr W. Legrand, Notes sur le culte de $S$. Poppon, abbé de Stavelot, dans Chronique Archéologique du Pays de Liège, t. XXIII, 1942, p. 34-48 et t. XXIV, 1943, p. 1-18 et 25-44.

69. A. Vauchez, La spiritualité du Moyen Âge occidental (VIII-XIII siècle), nv. éd., Paris, 1994, p. 78-95.

70. G. Despy, Les chartes privées de l'abbaye de Stavelot pendant le haut Moyen Âge (748-991), dans Le Moyen Age, t. LXII, 1956, p. 275.

71. Dom Daniel Misonne a précisé le terme "restauration" en en distinguant trois types: la réforme proprement dite qui consiste à rendre sa "forme " première à une institution décadente, la rénovation, et la $r e$ - création que nous expliquons ci-après (D. Misonne, La restauration monastique du $\mathrm{XIX}^{e}$ siécle. Questions de méthodologie, dans Rev. bén., t. LXXXIII, 1973, p. 34). Ces définitions proposées par Dom Misonne nous paraissent excellentes, même si, par facilité de langage, nous avons continué à employer le terme de " réforme " qui, soulignons-le bien, est abusif. 
permettait sans aucun doute aux religieux de se donner tout entiers à l'opus Dei ${ }^{72}$.

Au cours des $x^{e}$ et $x^{e}{ }^{e}$ siècles, l'abbaye va subir l'influence de deux mouvements monastiques profonds issus respectivement de Gorze et de Saint-Vanne de Verdun. Avec Gorze, on peut parler de "re-création " ou "résurrection» de l'abbaye: on repart à zéro, et des hommes nouveaux dans des situations nouvelles restaurent une vie monastique pratiquement disparue. Avec Saint-Vanne, on parlera de "rénovation ", qui n'implique pas spécialement une décadence mais qui est un réajustement par rapport à un programme conçu selon des vues différentes.

Enfin, à Malmedy, Erlebald fonde une léproserie, sans doute après s'être occupé à Stavelot de l'hôpital, sous l'abbatiat de son frère. Décidément l'action de l'ancien coûtre de l'abbaye s'exerce non seulement à propos des reliques mais aussi sur les revenus monastiques qui relèvent de la compétence de son ancien office. Tout se passe naturellement comme si, une fois devenu abbé, il se souvenait des problèmes à résoudre auxquels il fut confronté lorsqu'il était coûtre.

Erlebald s'est préoccupé directement et personnellement du trésor des reliques de son abbaye ${ }^{73}$. Sa dévotion aux reliques est manifeste à travers la documentation que nous avons rassemblée ${ }^{74}$. Cette dévotion

72. Ph. Schmitz, Histoire de l'Ordre de Saint-Benoît, Maredsous, 1948, t. I, p. 147; A. Dierkens, Abbayes, op. cit., p. 332 n. 2 et 335 sv,; M. Parisse, Noblesse et monastères en Lotharingie du $I X^{e}$ au $X I^{e}$ siecle, dans Monastische Reformen im 9. und 10. Jahrhundert, éd. par R. KotтJe \& H. Maurer, Sigmaringen, 1989 (Vorträge und Forschungen, t. XXXVIII), p. 184; M. MARgue, Aspects politiques de la "réforme * monastique en Lotharingie. Le cas des abbayes de Saint-Maximin de Trèves, de StavelotMalmedy et d'Echternach, dans Rev. bén., t. XCVIII, 1988, p. 31-32; P. BEcker, Monastische Reformstadien im trierischen und lothringischen Raum, gesehen aus der Perspeklive von Sankt Eucharius (11.-13. Jahrhundert), dans Échanges religieux \& intellectuels $d u X^{e}$ au $\mathrm{XIII}^{e}$ siècles en Haute et en Basse-Lotharingie. Actes des $5^{e}$ Journées Lotharingiennes, 21-22 octobre 1988, Luxembourg, Publicalions de la Section Historique de I'Institut Grand-Ducal Luxembourgeois, 1991, p. 135, et R. Scmieffer, Consuetudines monasticae und Reformforschung, dans Deutsches Archiv, t. XLIV, 1988, p. 161-169.

73. Notre article Erlebald $(\dagger 1192)$, gardien des reliques de Stavelot-Malmedy, dans Le Moyen Age, t. XC, 1984, p. 375-382.

74. Nous avons surpris l'action d'Erlebald à Malmedy et à Lierneux en 1185, ce qui enrichit sa notice hingraphique, bien sèche par rapport à celle de son illustre frère. Arant son accession à l'abbatiat, il apparaît témoin à un acte de 1146 , expressément qualifié de custos Stabulensis (HR 183); 1146, c'est-à-dire un an après la consécration du buste reliquaire du pape Alexandre, dont on retrouve aussi des reliques à Lierneux. Aussi, derrière le mécénat artistique de Wibald, extériorisation exceptionnelle de sa dévotion aux saints, nous ne pouvons plus négliger la personnalité de son frère Erlebald, directement préoccupé du trésor des reliques des monastères, une préoccupation qu'il conservera tout au long de son abbatiat.

La dévotion exceptionnelle d'Erlebald pour saint Remacle est sans doute héritée de son frère; il prolonge en effet le développement du culte du saint patron. En 1187 on trouve mention d'une relique de saint Remacle à Schöntal en Suisse et une autre à la même époque dans la boîte à reliques de Momalle, sans pouvoir, bien entendu, préten- 
n'est extraordinaire ni pour l'époque ni pour le milieu monastique dont est issu Erlebald; elle s’inscrit dans ce vaste mouvement international d'intérêt multiforme pour les saints et leurs reliques ${ }^{75}$, où l'on peut isoler de nombreuses personnalités comme Gérard de Brogne ${ }^{76}$, Richard de Saint-Vanne, Wibald de Stavelot ou plus tard Guillaume de Ryc$\mathrm{kel}^{77}$.

Cette préoccupation d'Erlebald pour le culte des reliques se double d'un souci particulier pour le culte des morts.

$\mathrm{Au}$ retour d'une ambassade, le 19 juillet 1158, Wibald décéda à Bitolj-Bitola (République de Macédoine). L'année suivante, son frère Erlebald devenu abbé de Stavelot-Malmedy, fit rapatrier sa dépouille mortelle qui fut inhumée le 26 juillet 1159 à Stavelot au pied de l'autel majeur; la cérémonie est grandiose, rehaussée de la présence de plusieurs abbés et de celle de l'évêque de Liège Henri de Leez ${ }^{78}$. De plus, Erlebald fonde son propre anniversaire à Stavelot, dotant la célébration de sa mémoire de divers biens et distribuant aux moines et aux pauvres des avantages en nature (HR 279). Déjà, le 21 mars 1182, il

dre que ces deux dons proviennent directement de Stavelot et sont contemporains d'Errebald. Toutefois, aux documents que nous avons rassemblés à propos de la boìte à reliques de Momalle, ca. 1182 , cfr: Deux reliquaires historiques (XI et $X I I^{e}$ siécles) conservés à Liège, dans Bulletin de la Société Nationale des Antiquaires de France, 1990 , p. 368-377 on ajoutera la présence en 1183 de Petrus de Moumale, commanditaire de l'œuvre, à la ratification par l'archidiacre Thierry de Liège de la cession de l'église paroissiale de Stavelot et de ses chapelles par Erlebald au monastère (HR 273), une occasion de plus de se procurer des reliques. En 1183, l'Inventio reliquiarum S. Eligii rapporte le pèlerinage d'un sainteur de Saint-Éloi de Noyon aux sources SaintRemacle à Stavelot (Analecta Bollandiana, t. IX, p. 432).

75. Cfr notre étude Les routes de la foi en pays mosan. Sources, méthode et problématique $\left(I V^{e}-X V^{e}\right.$ siecles), dans Feuillets de la Cathédrale de Liège, n' 18-20, 1995.

76. D. Misonne, Gérard de Brogne et sa dévotion aux reliques, dans Sacris Erudiri, t. XXV, 1982, p. 1-26.

77. Cfr notre article A Saint-Trond, un import-export de reliques des Onze Mille Vierges au X ${ }_{11}{ }^{e}$ siècle, dans Bulletin de la Société Royale Le Vieux-Liège, t. XII, no 252-253,
1991, p. 209-228.

78. Cuius [Wibaldi] ossa cum post annum et dies sex a fidelibus suis iussu serenissimi imperatoris Frederici primi ad nos relata fuissent, 7 Kal. Augusti a domno Henrico Leodiensi episcopo cum aliquantis abbatibus et magna frequentia cleri et populi, non sine magno dolore et luctu fratrum suorum venerabiliter sepulture sunt tradita ante altare beati Petri et Remacli, in loco qui est inter chorum et gradus cancelli, quatinus huius exemplo successores eius accensi res ecclesie et fratrum sibi creditas non distrahere vel alienare, set conservare et augere, imitatores eius facti, studeant. Manuscrit du $\mathrm{xv}^{\mathrm{e}}$ siècle retranscrit en partie dans Monumenta Corbeiensia, éd. Ph. JAFFe, Berlin, 1864 (Bibliotheca Rerum Germanicarum, t. I), p. 607 et repris par François Laurenty (IDEM, p. 608); cfr JAKoBI, op. cit., p. 188. Jusqu'à la dernière guerre, on conservait au Musée Curtius à Liège une pierre commémorative de l'ensevelissement en ce lieu du prélat, cfr N. W'EerTs, dans Cahiers de l'Institut Archéologique Liégeois, t. III, Liège, 1985, n ${ }^{\circ} 150$ p. $203-204$. 
avait concédé plusieurs biens pour la célébration d'un office funèbre pour son frère Wibald; après sa mort, il devait lui-même être adjoint à cette intention (HR 272). Mais, au fait, de quelle maladie est mort Wibald, et dans quelles circonstances exactes? Pouvoir répondre à cette question permettrait peut-être de sonder davantage encore les motivations profondes de son frère.

\section{Liege}

Philippe George 